\title{
Marine compounds inhibit growth of multiple myeloma in vitro and in vivo
}

\author{
Normann Steiner ${ }^{1,2}$, Domenico Ribatti4,5, Wolfgang Willenbacher ${ }^{2,7}$, Karin Jöhrer ${ }^{3}$, \\ Johann Kern ${ }^{1,7}$, Christian Marinaccio ${ }^{4}$, Miguel Aracil ${ }^{6}$, Luis F. García-Fernández ${ }^{6}$, \\ Guenther GastI ${ }^{2}$, Gerold Untergasser ${ }^{1,3, *}$ and Eberhard Gunsilius ${ }^{1,2, *}$ \\ ${ }^{1}$ Laboratory for Tumor Biology \& Angiogenesis, Innsbruck Medical University, Innsbruck, Austria \\ ${ }^{2}$ Department of Internal Medicine V, Innsbruck Medical University, Innsbruck, Austria \\ 3 Tyrolean Cancer Research Institute, Innsbruck, Austria \\ ${ }^{4}$ Department of Basic Medical Sciences, Neurosciences, and Sensory Organs, University of Bari Medical School, Bari, Italy \\ ${ }^{5}$ National Cancer Institute "Giovanni Paolo II", Bari, Italy \\ ${ }^{6}$ PharmaMar R\&D, Colmenar Viejo, Madrid, Spain \\ 7 Oncotyrol GmbH, Innsbruck, Austria \\ * These authors contributed equally to this work \\ Correspondence to: Eberhard Gunsilius, email: eberhard.gunsilius@i-med.ac.at \\ Keywords: Marine drugs, Angiogenesis, Multiple Myeloma, CAM, Xenografts \\ Received: December 02, $2014 \quad$ Accepted: January 15, $2015 \quad$ Published: January 31, 2015
}

This is an open-access article distributed under the terms of the Creative Commons Attribution License, which permits unrestricted use, distribution, and reproduction in any medium, provided the original author and source are credited.

\section{ABSTRACT}

Purpose: The prognosis of patients with multiple myeloma (MM) is still dismal despite recent improvements achieved by introducing new therapeutic agents. However, there remains an urgent need for progress in myeloma drug development. We here show that novel marine-derived compounds can exert potent anti-myeloma activity.

Experimental Design: Nine marine-derived compounds were applied at low $\mathrm{nM}$ concentrations (0.1-100 nM) to MM cell lines (OPM-2, NCI-H929, U266, RPMI-8226), to primary human myeloma cells and to peripheral blood mononuclear cells. Apoptosis was determined by flow cytometry. In addition, eGFP-transgenic MM cell lines growing with mesenchymal cells from bone marrow were used to visualize tumors by fluorescence stereomicroscopy. Anti-myeloma activities were studied in vitro in 3D spheroids and in vivo in myeloma xenografts on chicken embryos. Tumor size was analyzed by measuring GFP content with a GFP ELISA. Anti-angiogenic activities of compounds were tested in an in vivo gelatin sponge assay with conditioned media from primary bone marrow-derived endothelial cells.

Results: We identified a subset of marine compounds with strong anti-myeloma activity in vitro and in vivo. Moreover, some of the compounds inhibited myelomarelated angiogenesis in the in vivo gelatin sponge assay. They merit further drug development to improve treatment options for MM.

\section{INTRODUCTION}

Multiple myeloma (MM) is the second most frequent hematological malignancy [1], a malignant B-cell neoplasm with rising prevalence that causes considerable morbidity, mortality and health care expenditures $[2$, 3]. Although the last decade has seen considerable improvement in overall and progression-free survival due to the introduction of new drugs as proteasome inhibitors like bortezomib and carfilzomib and immunomodulatory agents including thalidomide, lenalidomide and pomalidomide [4, 5], in most cases MM remains an incurable disease with inevitable disease progression due to clonal evolution, development of drug resistance and fatal outcome [4]. 
Table 1: Induction of apoptosis by marine-derived compounds in MM cell lines ( $\%$ of living cells)

\begin{tabular}{|c|c|c|c|c|c|c|c|c|c|c|c|c|}
\hline $\begin{array}{l}\text { Cell } \\
\text { type }\end{array}$ & $\begin{array}{l}\text { Concen- } \\
\text { tration }\end{array}$ & Con. & Bortezomib & Plitidepsin & Lamellarin D & Elisidepsin & ES-285 & Zalypsis & PM00113 & PM01215 & PM02781 & Thiocoraline $\mathrm{A}$ \\
\hline \multirow{2}{*}{ OPM-2 } & $10 \mathrm{nM}$ & \multirow{2}{*}{80} & $49 *$ & 78 & 73 & 77 & 76 & $44^{*}$ & $39 *$ & 77 & 74 & 76 \\
\hline & $100 \mathrm{nM}$ & & $44 *$ & $52^{*}$ & $53^{*}$ & 78 & 78 & $42^{*}$ & $39^{*}$ & $62^{*}$ & $39^{*}$ & $62^{*}$ \\
\hline \multirow{2}{*}{ U266 } & $10 \mathrm{nM}$ & \multirow{2}{*}{80} & $22^{*}$ & 75 & 77 & 76 & 76 & $40^{*}$ & $42^{*}$ & 71 & 71 & 70 \\
\hline & $100 \mathrm{nM}$ & & $20^{*}$ & $52^{*}$ & 71 & 76 & 76 & $38^{*}$ & $39^{*}$ & $59 *$ & $54^{*}$ & $62^{*}$ \\
\hline NCI- & $10 \mathrm{nM}$ & \multirow{2}{*}{73} & $6^{*}$ & 67 & 70 & 69 & 73 & $52 *$ & $41^{*}$ & 69 & 66 & $55^{*}$ \\
\hline H929 & $100 \mathrm{nM}$ & & $5^{*}$ & $6^{*}$ & $36^{*}$ & 68 & 72 & $7 *$ & $7^{*}$ & $10^{*}$ & 9* & 9* \\
\hline
\end{tabular}

MM cell lines (OPM-2, U266, NCI-H929) were incubated with increasing concentrations of drugs for $24 \mathrm{~h}$ and then analyzed by flow cytometry. Annexin- $\mathrm{V}^{\text {neg }}$ and $7 \mathrm{AAD}^{\text {neg }}$ cells were defined as viable. Data were calculated from triplicate results for each drug; $10 \mathrm{nM}$ or $100 \mathrm{nM}$. Highlighted numbers indicate significant killing of myeloma cell lines. Stars indicate $\mathrm{p}$ values $<0.05$. Con.: control.

MM is a highly heterogeneous malignancy characterized by complex primary and secondary cytogenetic and molecular aberrations with varying clinicopathological features and disease course $[6,7]$. Due to intraclonal and interclonal tidings MM cells constitute a "moving therapeutic target" [8]. Therefore, new drugs might supplement the current therapeutic armamentarium by targeting putative driver genes and signaling pathways in MM cells and affecting the myeloma stromal niche in the bone marrow.

In this regard, the marine environment with its large biodiversity constitutes a rich source of potential therapeutic compounds. Systematic high-throughput screening has identified the antitumor potential of a variety of marine-derived compounds with significant biological activities in vitro and in vivo. Several marine lead compounds are currently under development as cancer therapeutics. Plitidepsin (Aplidin), an anticancer agent originally isolated from the tunicate Aplidium albicans and currently obtained by total synthesis, is being tested in a phase III trial in patients with relapsed or refractory multiple myeloma (registered with www.clinicaltrials. gov, number NCT01102426). At the molecular level, Plitidepsin is a potent inducer of apoptosis. This effect has been related to its interaction with eEF1A and the generation of early oxidative stress, which triggers the sustained activation of JNK and p38 MAPKs [9] and leads to endoplasmic reticulum (ER) stress and subsequent unfolded protein response (UPR) activation (Drug Discovery Area, PharmaMar S.A.U., unpublished data).

In this study, we assayed a selected set of marine substances with respect to anti-myeloma activities in vitro and in vivo against standard anti-myeloma compounds, such as bortezomib, and substances in advanced development like Plitidepsin as reference.

\section{RESULTS}

\section{Screening for compounds with anti-myeloma activity using myeloma cell lines in vitro}

Marine compounds were tested for their ability to induce apoptosis in human MM cell lines. For this purpose, cell viability was measured by flow cytometry $24 \mathrm{~h}$ after incubation with increasing concentrations of test compounds. Most compounds showed anti-myeloma activity in vitro when tested at concentrations of $100 \mathrm{nM}$ in commercially available human MM cell lines growing under standard culture conditions (Table 1, Supplementary Figure 1). Plitidepsin, Zalypsis, PM00113, PM01215, PM02781 and Thiocoraline A induced significant apoptosis in all MM cell lines at $100 \mathrm{nM}$. A strongly dose-dependent effect was seen with Zalypsis, PM00113 and Thiocoraline A; these compounds were even effective at a concentration of $10 \mathrm{nM}$ in one or more cell lines inducing apoptosis like the reference compound bortezomib. Notably, at 100 nM concentration compounds Plitidepsin, Zalypsis, PM00113 and Thiocoraline A also induced apoptosis of peripheral blood mononuclear cells (PBMNC) from five healthy donors (Supplementary Figure 2A) comparable to bortezomib $100 \mathrm{nM}$ (apoptotic rate increased from $11.3 \pm$ 3.2 in control cells to $28.0 \pm 4.3 \%$ in bortezomib-treated cells, data not shown).

\section{Screening for compounds with anti-myeloma activity using bone marrow aspirates of patients with multiple myeloma}

Anti-myeloma activity of the marine compounds was examined in vitro in ten samples of primary human multiple myeloma cells. Clinical data of all patients are 
A
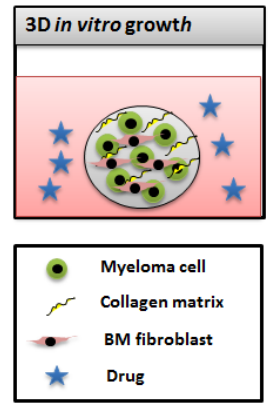

B
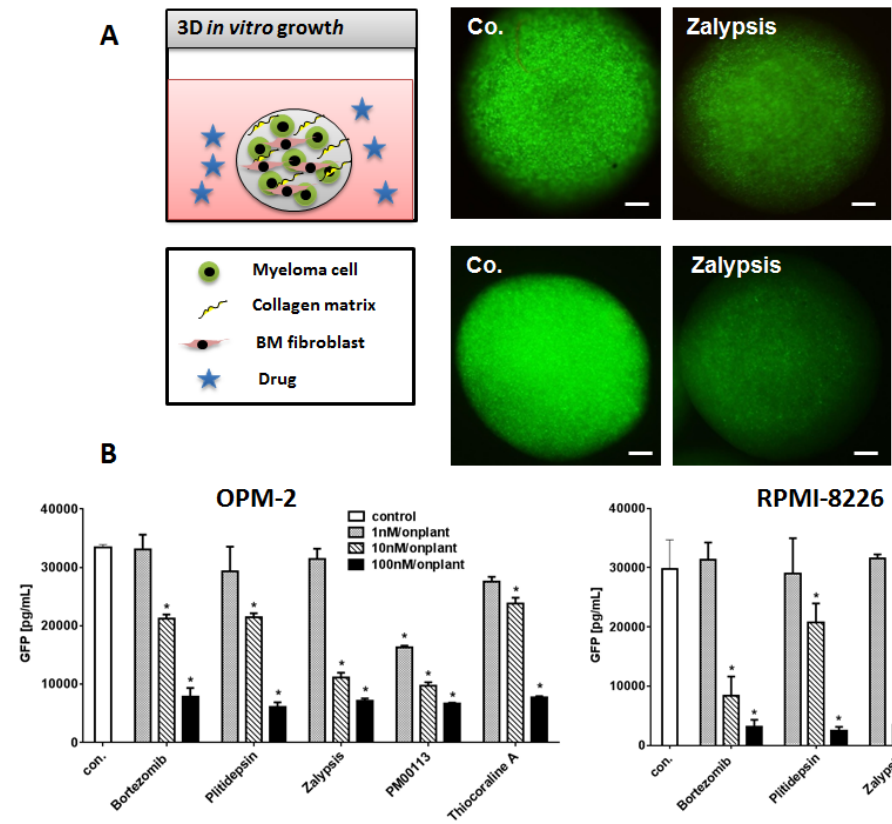

OPM-2

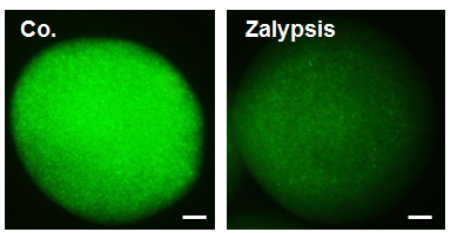

RPMI-8226

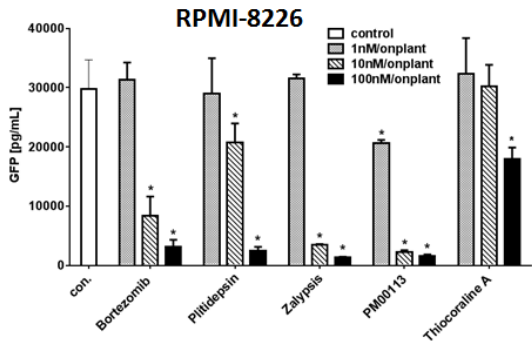

Figure 1: 3D Multiple Myeloma Model. (A) Human multiple myeloma cell lines were lentivirally transfected and selected to stably express eGFP. OPM-2 $2^{\text {eGFP }}$ and RPMI- $8226^{\text {eGFP }}$ cells were cultivated in hanging drops with primary human bone marrow mesenchymal cells and collagen type I as extracellular matrix component. Spheroids were incubated with culture medium (co.), and respective concentrations (1-100 nM) of all target compounds effective on primary MM cells. MM cell lines were grown for $72 \mathrm{~h}$ in spheroids and photographed daily using a stereo-fluorescence microscope. Bars indicate $500 \mu \mathrm{m}$. (B) Single spheroids were homogenized in lysis buffer and subsequently measured in a GFP ELISA. GFP concentrations of single spheroids were calculated ( $\mathrm{n}=6, \mathrm{Mean} \pm \mathrm{SEM}$ ). Stars indicate $\mathrm{p}$ values $<0.05$. Con.: control.

A
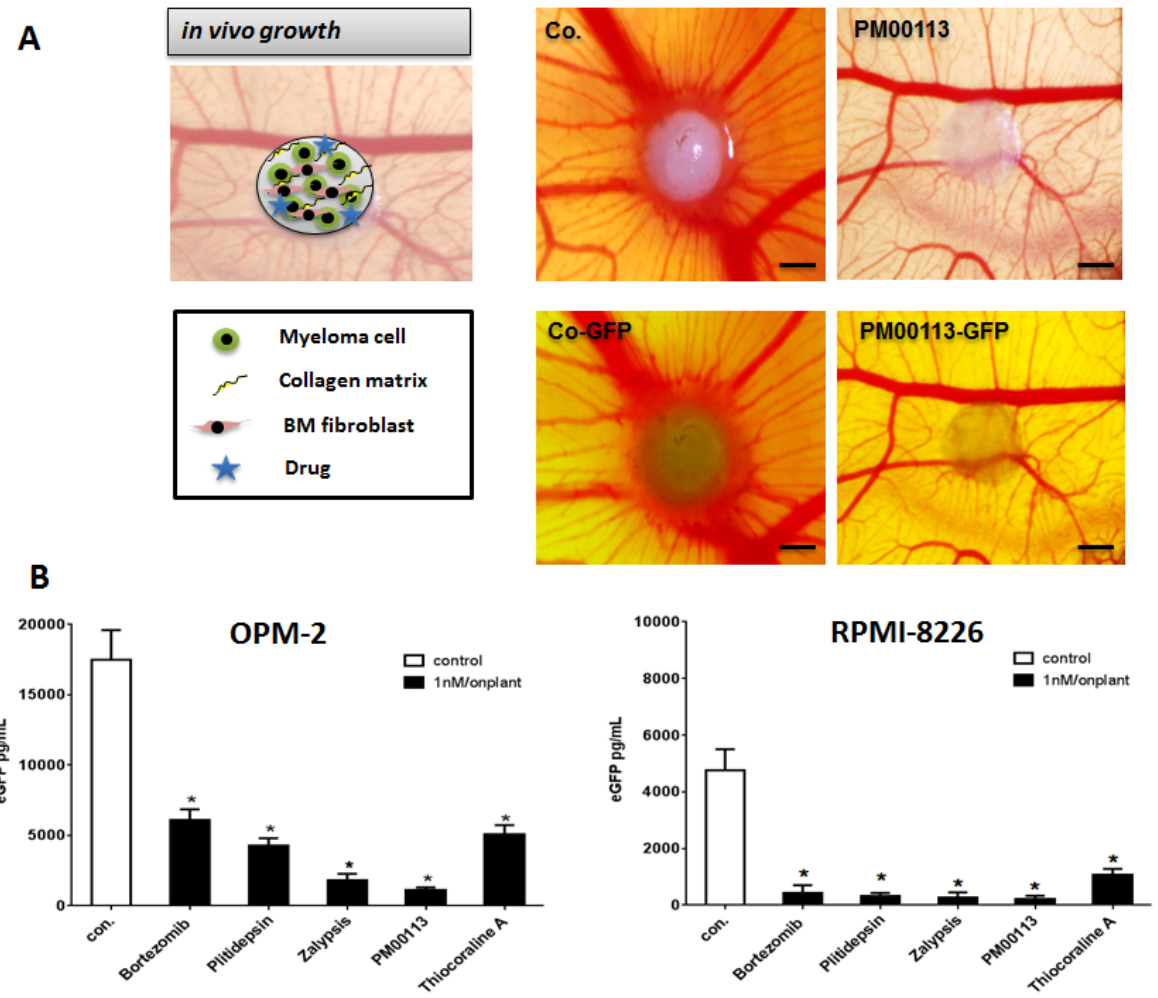

Figure 2: Multiple Myeloma Xenograft Model. (A) OPM-2 ${ }^{\text {GFP }}$ and RPMI-8226 $6^{\text {eGFP }}$ cells were mixed with primary human bonemarrow mesenchymal cells, collagen type I as extracellular matrix component and with $1 \mathrm{nM}$ of the respective target compound and grafted on the chorioallantoic membrane of chicken embryos $(\mathrm{n}=3)$. After five days tumors can be visualized by eGFP expression. In grafts, all drugs showed significant lower fluorescence than the control. Bars indicate $500 \mu \mathrm{m}$. (B) Single MM xenografts were excised and homogenized in lysis buffer and subsequently measured using a GFP ELISA. GFP concentrations of single tumors were calculated ( $\mathrm{n}=6$, Mean $\pm \mathrm{SEM}$ ). Stars indicate $\mathrm{p}$ values $<0.05$. con.: control. 
Table 2: Induction of apoptosis by marine-derived compounds in primary myeloma cells and PBMNC (\% of living cells)

\begin{tabular}{|c|c|c|c|c|c|c|c|c|c|c|c|}
\hline Patient & Con. & Bortezomib & Plitidepsin & Lamellarin D & Elisidepsin & ES-285 & Zalypsis & PM00113 & PM01215 & PM02781 & Thiocoraline A \\
\hline MM\#1 & 79 & $12 *$ & 73 & 81 & 77 & 82 & $15^{*}$ & $18^{*}$ & 77 & 83 & $65^{*}$ \\
\hline MM\#2 & 79 & $59 \%$ & 75 & 72 & 74 & 82 & $62 *$ & $67 *$ & $66^{*}$ & 73 & $62 *$ \\
\hline MM\#3 & 82 & 77 & $51^{*}$ & 83 & 85 & 81 & $16^{*}$ & $15^{*}$ & 70 & 70 & 76 \\
\hline MM\#4 & 67 & $6^{*}$ & $46^{*}$ & 63 & 65 & $53^{*}$ & $10 *$ & 9* & 61 & 58 & 58 \\
\hline MM\#5 & 89 & $20 *$ & 74 & 86 & 88 & 86 & $5^{*}$ & $5^{*}$ & 76 & $69 *$ & 82 \\
\hline MM\#6 & 80 & $12 *$ & $26^{*}$ & 72 & 73 & 79 & $5^{*}$ & $6^{*}$ & $51 *$ & $54 *$ & $69 *$ \\
\hline MM\#7 & 82 & $50 *$ & 80 & 82 & 82 & 82 & 83 & 80 & 79 & 78 & 74 \\
\hline MM\#8 & 72 & $29 *$ & $18^{*}$ & 74 & 70 & 71 & $16^{*}$ & $14 *$ & 64 & 72 & $58^{*}$ \\
\hline MM\#9 & 66 & $5^{*}$ & 64 & 68 & 67 & 64 & $18^{*}$ & $10^{*}$ & 65 & 60 & 60 \\
\hline MM\#10 & 74 & $27^{*}$ & $39^{*}$ & 74 & 65 & 72 & $18^{*}$ & $24^{*}$ & 65 & 70 & $56^{*}$ \\
\hline PBMNC & 81 & 76 & 83 & 85 & 84 & 82 & 82 & 82 & 81 & 82 & 80 \\
\hline
\end{tabular}

Exemplary experimental data from multiple myeloma patients $(\mathbf{n}=\mathbf{1 0})$. MM cells were defined as $\mathrm{CD} 38^{\text {high }}$ and $\mathrm{CD} 45^{\text {low }}$. Peripheral blood mononuclear cells (PBMNC) from donors served as control for toxicity in primary cells. Data from triplicate results of 10 $\mathrm{nM}$ concentrations of each drug (S1-S9). Mean of triplicate results, stars indicate $\mathrm{p}$ values $<0.05$. Con.: control. Highlighted compounds efficiently killed primary MM cells at concentrations of $10 \mathrm{nM}$, but not primary PBMNC in at least five patient samples. These compounds were used for further studies in MM in vitro and in vivo models.

provided in Supplementary Table 1. Whole bone marrow, containing MM cells, normal hematopoietic and stromal cells of the microenvironment, was cultivated in vitro and incubated with increasing concentrations of target compounds. After $24 \mathrm{~h}$ primary MM cells were identified by expression of $\mathrm{CD} 45^{\text {low }}$ and $\mathrm{CD} 38^{\text {high }}$ in flow cytometry with gating for viable myeloma cells (annexin- $V^{\text {neg }}$ $\left./ 7 \mathrm{AAD}^{\text {neg }}\right)$. In contrast to commercially available MM cell lines, primary patient MM cells cultivated in context with the respective cells of their microenvironment were remarkably more sensitive to applied drugs (Table 2, Supplementary Figure 3). In fact, most of the drugs effectively induced apoptosis even at a concentration of 10 nM. In detail, Plitidepsin, Zalypsis, PM00113 and Thiocoraline A applied at a concentration of $10 \mathrm{nM}$ showed significant anti-myeloma activity against primary MM cells in vitro. PM01215 and PM02781 at $10 \mathrm{nM}$ demonstrated apoptotic activity only in individual patients (Table 2) and thus were excluded from further analysis. MM cells from one patient (MM\#3, clinically refractory to bortezomib, see Table 2) were not sensitive to bortezomib at all, but were readily killed in vitro with marine compounds. MM cells from patient MM\#1 (newly diagnosed MM), MM\#5 (progressive disease with plasma cell leukemia), MM\#6 (progressive disease), MM\#9 (progressive disease) and MM\#10 (progressive disease), all five considered high-risk patients, showed a significant apoptosis response when treated with two to four of the marine-derived compounds. All but one patient sample (MM\#7; high-risk myeloma, progressive disease) were sensitive to marine-derived compounds. With regard to peripheral blood mononuclear cells (PBMNC, $\mathrm{n}=5$ ) all tested compounds displayed no significant apoptotic effects at concentrations of $10 \mathrm{nM}$, as determined by staining for annexin- $\mathrm{V}^{\text {neg }} / 7 \mathrm{AAD}^{\text {neg }}$ and flow cytometric analysis (Table 2, Supplementary Figure 2A).

Based on these findings made in primary MM cell cultures, the four compounds Plitidepsin, Zalypsis, PM00113 and Thiocoraline A, effective at $10 \mathrm{nM}$, were used for further analysis in $3 \mathrm{D}$ in vitro and in vivo assays of MM cells with the respective mesenchymal microenvironment.

\section{In vitro analysis of selected marine compounds in 3D in vitro multiple myeloma spheroid assays}

Due to the limitations of culturing primary human MM cells in vitro we established a $3 \mathrm{D}$ in vitro culture model of human MM cell lines using a collagen extracellular growth matrix and supportive primary human mesenchymal cells from bone marrow. MM cell lines transgenic for eGFP allow visualization and quantification of $\mathrm{MM}$ tumor mass after $3 \mathrm{D}$ growth in spheroids (Figure 1A). Both MM cell lines OPM-2 ${ }^{\text {eGFP }}$ and RPMI-8226 ${ }^{\text {eGFP }}$ were cultivated for three days in the presence of increasing concentrations (1-100 nM) of the respective target compounds, and tumors were visualized by GFP expression (Figure 1A). All target compounds significantly inhibited $\mathrm{MM}$ growth at concentrations of 10 and $100 \mathrm{nM}$ in OPM-2 $2^{\text {eGFP }}$ and RPMI-8226 $6^{\text {eGFP }}$ spheroids.

Tumor cell mass was quantified after spheroids 
were homogenized and eGFP content was measured with a GFP-ELISA (Figure 1B). With regard to the applied drug molarity, Zalypsis and PM00113 were the most potent inhibitors in OPM-2 ${ }^{\text {eGFP }}$ and RPMI-8226 $6^{\mathrm{eGFP}} 3 \mathrm{D}$ spheroids, reaching or even exceeding the anti-myeloma activity of bortezomib. Noteworthy, in MM 3D spheroids none of the marine drugs induced apoptosis in mesenchymal cells, even at concentrations of $100 \mathrm{nM}$ (data not shown). These data confirm our analysis of apoptosis induction in standard culture of primary human mesenchymal cells derived from bone marrow and exposed to marine compounds, which cells were significantly more resistant to apoptosis than were MM cells (Supplementary Figure 2B).

\section{In vivo analysis of selected marine compounds using myeloma xenografts in chicken embryos}

EGFP-transgenic myeloma cells (OPM-2 ${ }^{\text {eGFP }}$ and RPMI-8226 ${ }^{\text {eGFP }}$ ) together with ex vivo-derived human bone-marrow mesenchymal cells were embedded in collagen type I as extracellular matrix component. Test substances were applied topically at $1 \mathrm{nM}$ concentration. These "onplants" were grafted on the chorioallantoic membrane of chicken embryos (using six replicates). After five days, MM xenografts formed tumors that could be visualized by eGFP expression (Figure 2A). Compared to controls (0.1\% DMSO), all analyzed target compounds inhibited growth of MM cells in xenografts resulting in less green-fluorescent MM tumor cell mass (Figure $2 A)$. Single MM xenografts ( $n=6 /$ group) were excised, homogenized and thereafter measured by GFP ELISA. All tested compounds significantly reduced myeloma cell

\section{A}

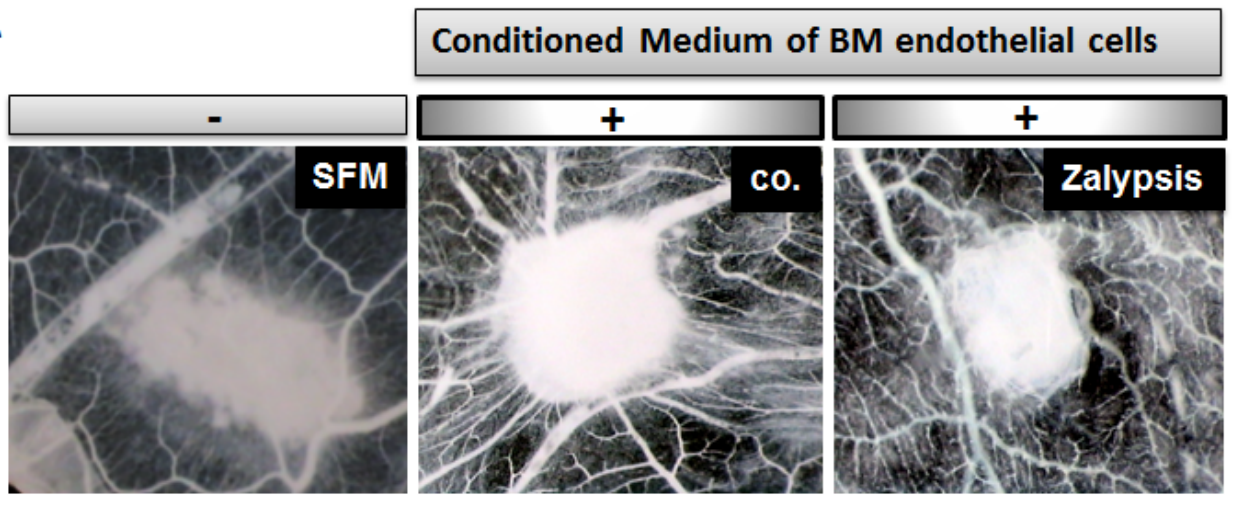

B

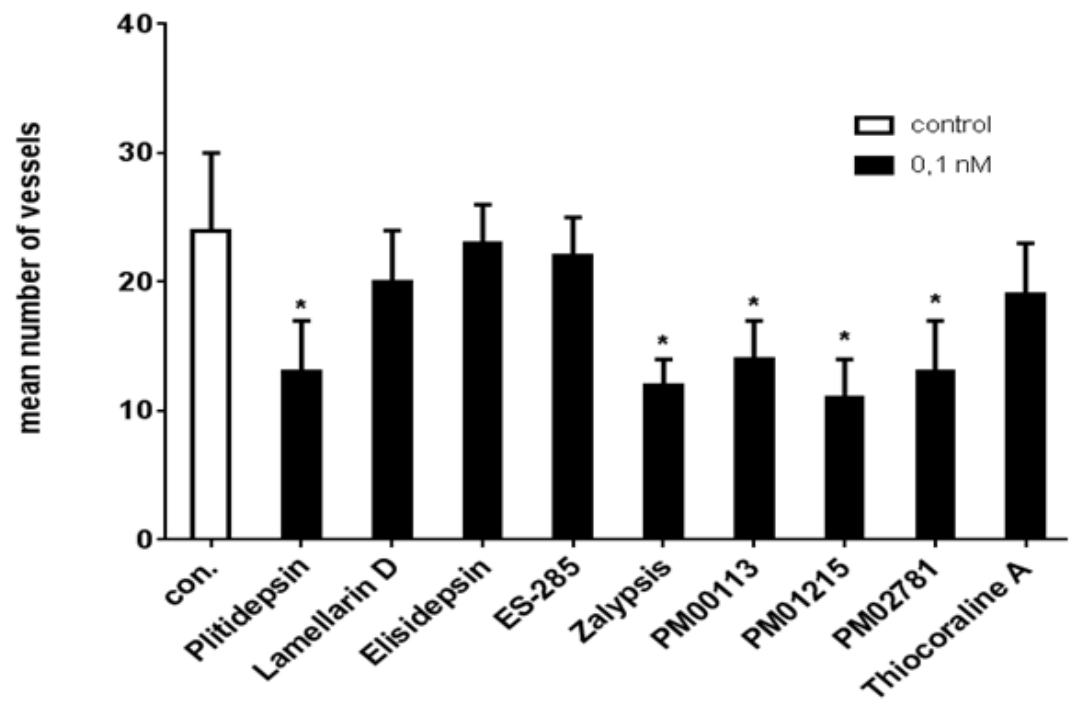

Figure 3: Anti-angiogenic activities of marine-derived compounds in the gelatin sponge assay. (A) CAMs were incubated with gelatin sponges loaded with SFM (left) and with conditioned medium of multiple myeloma-derived endothelial cells either alone (co.) or supplemented with $0.1 \mathrm{nM}$ of marine-derived compounds (drug). Note the decreased number of blood vessels. Images were acquired with a stereomicroscope (magnification, x 50). (B) A set of marine-derived compounds tested at $0.1 \mathrm{nM}$ demonstrated anti-angiogenic activity in response to conditioned media of endothelial cells deriving from patients suffering from multiple myeloma $(\mathrm{p}<0.05)$. The mean number of vessels in the control was $24 \pm 6$. Stars indicate $p$ values $<0.05$. 
mass in a manner similar to that observed for bortezomib (Figure 2B). Again Zalypsis and PM00113 were the most potent inhibitors of myeloma growth in vivo, exceeding even the anti-myeloma activity of bortezomib.

In addition, we observed that revascularization of xenografts and microvessel sprouting adjacent to grafts were significantly reduced by marine-derived compounds (Figure 2A).

\section{Anti-angiogenic activities of target compounds in vivo in the gelatin sponge assay}

To investigate whether marine-derived compounds could also inhibit myeloma-related angiogenesis, we tested all compounds in the in vivo CAM assay. Sponges soaked with conditioned medium of bone marrow-derived endothelial cells from patients with MM were grafted with compounds S1-S9 on chicken chorioallantoic membranes (CAMs). CAMs implanted with a gelatin sponge together with conditioned medium were surrounded by a rim of newly formed capillaries converging radially toward the sponge in a "spoked-wheel" pattern (mean number of vessels: $24 \pm 6$; Figure 3A; with serum-free medium: mean number of vessels: $9 \pm 2$ ). Marine-derived compounds applied at low $\mathrm{nM}$ concentrations markedly inhibited the formation of capillaries (Figure 3A). Addition of compounds at even a concentration of $0.1 \mathrm{nM}$ resulted in a significant inhibition of MM endothelial cellinduced angiogenesis for Plitidepsin, Zalypsis, PM00113, PM01215 and PM02781 (Figure 3B).

\section{DISCUSSION}

Recently, progression-free and overall survival rates in MM patients have been substantially improved by novel classes of therapeutics such as proteosome inhibitors and immunomodulatory drugs (IMIDs) $[4,5]$. Nevertheless, virtually all patients relapse and ultimately become resistant to therapy. Thus, scanning for new anti-myeloma drugs and development of combinatorial therapeutic strategies are essential to further improve the outcome of patients with MM. A rapidly growing field of interest is the development of novel drugs that directly target myeloma cells and/or modify the respective microenvironment of MM cells in the bone marrow.

This study evaluated new marine-derived compounds for their anti-myeloma activity in vitro and in vivo including direct and indirect effects on myeloma cells. A subset of a total of 9 compounds tested (Plitidepsin [S1], Zalypsis [S5], PM00113 [S6] and Thiocoraline A [S9]) demonstrated significant proapoptotic activity in primary human myeloma cells even at $10 \mathrm{nM}$ concentrations, comparable to bortezomib as a standard. In vitro primary human myeloma cells were more sensitive to the substances than were established myeloma cell lines. The same set of marine-derived drugs proved to be growth-inhibitory in human MM xenografts in vitro and in vivo. Plitidepsin blocks cell proliferation by arresting the cells in transition from G0/G1 to S phase and triggers apoptosis by inducing cytochrome $\mathrm{c}$ release and activating caspase 9 and 3 [10]. In addition, it also exerts antiangiogenic activity by reducing the secretion of VEGF and the expression of VEGF receptor-1 from MOLT-4 human leukemia cells in vitro [11]. Currently, this compound is undergoing clinical testing in a phase III trial for the treatment of relapsed/refractory MM (registered with www.clinicaltrials.gov, number NCT01102426). Our 3D in vitro co-culture data and in vivo xenograft data strongly support the development of Plitidepsin as an anti-myeloma drug.

Zalypsis is able to induce cell cycle arrest and apoptosis by provoking double-strand DNA breaks [12]. The strong anti-myeloma effect of this agent has already been described in MM cell lines resistant to standard anti-myeloma treatment [12]. This drug has already been suggested by Ocio and coworkers as one of the most powerful agents that have been evaluated against multiple myeloma [12]. Their findings were confirmed by our MM model systems and extended by analysis of MM primary cells. PM00113, an analog of Zalypsis, is considered to have a similar mode of action, i.e. DNA-binding and inducing double-strand breaks. This new marine-derived compound showed potent anti-myeloma effects at low $\mathrm{nM}$ concentrations in vitro on $\mathrm{MM}$ cell lines $(10 \mathrm{nM})$ as well as on primary human myeloma cells $(10 \mathrm{nM})$ and on GFP-transgenic myeloma-xenografts in vivo (1 nM). Thus, further development of the Zalypsis analog for MM is warranted.

Thiocoraline A is a marine-derived cytotoxic antibiotic. This compound causes an arrest in G1 phase and decreases the rate of $\mathrm{S}$ phase progression towards G2/M phases due to DNA polymerase- $\alpha$ inhibition [13]. In our MM cell lines and primary MM cells, Thiocoraline A showed potent inhibition of myeloma growth in a dosedependent manner.

For the purpose of future clinical drug development we conducted a more detailed analysis of the four marine agents effective against primary myeloma cells. Primary bone marrow-derived myeloma cells from MM patients even with high-risk cytogenetic features and progressive disease were remarkably sensitive to marinederived compounds. As an example, although patient \#3 was refractory to treatment with dexamethasone and bortezomib, myeloma cells from his bone-marrow were resistant to bortezomib in vitro but were readily killed by compounds Plitidepsin, Zalypsis and PM00113 even at low nanomolar concentrations (10 nM), suggesting the lack of cross-resistance between bortezomib and these marine-derived substances.

Zalypsis and PM00113 were the most potent inhibitors of myeloma growth, exceeding the anti- 
myeloma activity of bortezomib. Since our experiments showed no cross-resistance to bortezomib, synergies of these marine-derived compounds with established anti-MM drugs including bortezomib and IMIDs can be expected and should be evaluated.

Apart from directly affecting myeloma cells, some of the compounds (Plitidepsin, Zalypsis, PM00113) can also modify the myeloma-associated microenvironment, e.g. via the inhibition of myeloma cell-induced formation of new blood vessels. In vivo experiments using the CAM assay revealed significant suppression of myelomainduced neoangiogenesis close to xenografts and diminished revascularization of xenografts following exposure to marine agents, even at low concentrations $(0.1 \mathrm{nM})$.

Finally, to screen for drug toxicity in vitro we analyzed the proapoptotic activity of marine-derived compounds on normal blood mononuclear cells using flow cytometry. All four compounds with significant anti-myeloma activity in vivo also induced apoptosis of PBMNC and bone marrow-mesenchymal cells in vitro, but only at high drug concentrations $(100 \mathrm{nM})$. We thus conclude that the proapoptotic effects of selected marine compounds are dose-dependent and limited to neoplastic cells when these drugs are applied at drug concentrations of $10 \mathrm{nM}$ or less.

In conclusion, we identified a set of new marine compounds with strong anti-myeloma activity in vitro and in vivo, representing valid candidates for further preclinical and clinical development in myeloma. Our data indicate no cross-resistance of these drugs with bortezomib, suggesting that they could be used in combination with established anti-myeloma drugs.

\section{MATERIALS AND METHODS}

\section{Ethics statement}

Investigations have been conducted in accordance with the ethical standards and according to the Declaration of Helsinki and according to national and international guidelines and have been approved by the authors' institutional review board.

\section{Reagents}

All marine-derived compounds were synthesized by PharmaMar (Colmenar Viejo, Madrid, Spain), stocked in DMSO at a concentration of $1[\mathrm{mM}]$ and stored at $-20^{\circ}$ C. Bortezomib was purchased from Selleckchem and dissolved in DMSO (SIGMA Biochemicals) as a stock solution of $250 \mathrm{mM}$. Tunicamycin was purchased from Sigma. All compounds were freshly diluted in culture medium immediately before use.
Plitidepsin (Aplidin) [S1] is a depsipeptide discovered in the tunicate Aplidium albicans [14]. Lamellarin D [S2] was initially isolated from a marine mollusk of the Lamellaria sp. [15] and subsequently identified from ascidians [16]. The synthetic antitumor agent Elisidepsin [S3] is structurally related to Kahalalide $\mathrm{F}$, originally isolated from the Hawaiian marine mollusk Elysia rufescens [17]. The compound ES-285 [S4] was discovered in the sea mollusk Spisula polynyma [17]. Zalypsis [S5] is a synthetic alkaloid, related to the marine natural compound Jorumycin and the family of Renieramycins originally obtained from pacific mollusks and sponges $[18,19]$. Zalypsis has been shown to exert potent antimyeloma activity by inducing DNA double-strand breaks [12]. PM00113 [S6], an analog of Zalypsis, is suspected to have a mode of action similar to that of tetrahydroisoquinoline alkaloids. PM01215 [S7] and PM02781 [S8] are two novel synthetic analogs of Plitidepsin. Thiocoraline A [S9], a cytotoxic antibiotic, was originally isolated from the actinomycete Micromonospora marina, found in southeast Africa [20]. Chemical structures of all compounds are shown in Supplementary Figure 4.

\section{Cell culture}

The MM cell lines OPM-2 (ACC 50), U266 (ACC 9), NCI-H929 (ACC 163), and RPMI-8226 (ACC 402) were purchased 2012 directly from DSMZ (Germany), authenticated by us by flow cytometry (CD138+/CD38+) as well as by STR-profiling (data shown in Supplementary Table 2) and cultivated in RPMI1640 medium (Sigma Aldrich), supplemented with $10 \%$ bovine calf serum (Hyclone) and $100 \mathrm{IU} / \mathrm{mL}$ penicillin, $100 \mu \mathrm{g} / \mathrm{mL}$ streptomycin and $2 \mathrm{mM}$ glutamine (all PAA Laboratories $\mathrm{GmbH}$ ) in the presence of $5 \% \mathrm{CO}_{2}$ at $37^{\circ} \mathrm{C}$. OPM- 2 and RPMI-8226 cells were lentivirally transfected to express eGFP and propagated in the presence of blasticidin $(2.5 \mu \mathrm{g} /$ $\mathrm{mL}$, Invitrogen) before usage for in vivo experiments.

Peripheral blood mononuclear cells (PBMNC) were separated from heparinized blood from five healthy volunteers by Lymphoprep (PAA Laboratories $\mathrm{GmbH}$ ) gradient centrifugation, washed three times in Dulbecco's Phosphate-Buffered Saline (DPBS; Bio Whittaker), counted and resuspended in the above mentioned culture medium.

Human mesenchymal stem cells from bone marrow from three healthy donors were purchased from PromoCell. Cells were cultivated in MM cell line culture medium on uncoated plastic material.

Primary myeloma cells were obtained from heparinized bone marrow aspirates from patients with active MM ( $\mathrm{n}=10$; for details see Supplementary Table 1) following approval by the local ethics committee and the patients' written informed consent. Aspirates were cultivated in MM cell line culture medium in uncoated 
Falcon plastic flasks.

\section{Detection of cell viability by flow cytometry}

PBMNC and MM cells (cell lines and CD38 high / CD45 ${ }^{\text {low }}$ primary myeloma cells) were seeded into nutrient medium in 96-well plates (100,000 cells/ well). Marinederived drugs and bortezomib as reference substance were applied at concentrations of 0.1-100 nM. Cells were exposed to the various drugs for 24 hours in an atmosphere of $5 \% \mathrm{CO}_{2}$ at $37^{\circ} \mathrm{C}$. All experiments were done in triplicate. Cell immunostaining was performed with antibodies directed against human CD45 (labelFITC) and CD38 (label-PE) purchased from Miltenyi BD Biosciences.

Cell viability was determined by annexin V APC (Mab Tag) /7-AAD (7-amino-actinomycin D; Beckman Coulter) staining in a binding buffer consisting of $40 \mathrm{mM}$ $\mathrm{N}$-2-hydroxyethylpiperazine-N-2-ethanesulfonic acid, pH 7.4, $560 \mathrm{mM} \mathrm{NaCl}$, and $10 \mathrm{mM} \mathrm{CaCl}_{2}$. Stained cells were measured on the flow cytometer (FACSCanto ${ }^{\mathrm{TM}} \mathrm{II}$, BD Biosciences) and analyzed with the BD FACS DIVA software. Results were expressed as percentage of viable cells, i.e. $\%$ of living cells \pm standard error of the mean (SEM).

\section{Generation of eGFP-encoding lentiviruses}

The GFPmut1 chromophore is available in a codon-optimized version (eGFP) in the commercial vector pCMS eGFP (Invitrogen). The eGFP open reading frame with 3' UTR and polyadenylation signal was cloned by amplifying the fragment by means of KOD polymerase (Novagen) and specific forward (eGFP-for: 5tgcaaaaagcttgccacaac) and reverse primers (5 eGFP-rev: 5- ATTGTCTCATGAGCGGATAC).

The resulting PCR product was ligated into the pENTR11 Gateway vector (Invitrogen, Vienna, Austria), predigested with Xmn-I and Eco RV (NEB) and polished with Klenow. All amplified and purified pENTR11 vectors were control-sequenced for correct orientation and exclusion of incorporated mutations. pENTR 11 vectors were site-specifically recombined with the pLenti6-V5 DEST vector (Invitrogen) using the Gateway LR Clonase II Pus Enzyme Mix (Invitrogen). The resulting pLenti6 DEST vector with eGFP openreading frame was transformed and propagated in OneShot Stabl3 bacteria (Invitrogen). Lentiviruses were produced in HEK293FT cells by transfecting cells with the pDEST6 vectors and helper plasmid mix (ViraPower, lentiviral support kit) using Lipofectamine 2000 (both from Invitrogen). Lentiviral titers were determined by real time PCR and quantification of woodchuck hepatitis virus posttranscriptional response element expression (WPRE-for: 5-actgacaattccgtggtgtt; WPRE- rev: 5-agatccgactcgtctgagg). Cell lines were lentivirally transfected (pDEST6 eGFP) and stable cell lines selected with $2 \mu \mathrm{g} / \mathrm{mL}$ blasticidin (Invitrogen). Cell lines expressing the green reporter eGFP after two weeks of selection were named OPM-2 ${ }^{\text {GFP }}$ and RPMI- $8226^{\text {GGFP }}$.

\section{D myeloma spheroid model}

Transgenic MM cell lines (OPM-2 ${ }^{\text {eGFP }}$ and RPMI$8226^{\text {eGFP; }} 250,000$ per spheroid) were mixed into the collagen together with human mesenchymal stromal cells $(50,000$ cells/ spheroid $)$ or with the respective concentrations of compounds (1-100 nM). Aliquots of the collagen/cell mixture $(30 \mu \mathrm{l})$ were distributed over a paraffin film in a 12-well plate and allowed to polymerize for $30 \mathrm{~min}$ at $37^{\circ} \mathrm{C}$, generating so-called collagen spheroids. Thereafter, spheroids were overlaid with culture medium containing the respective concentrations of compounds (1-100 $\mathrm{nM})$ and cultivated for three days. After 72 hours of incubation at $37 \%$, the spheroids were documented with the Olympus SZX10 stereomicroscope (Olympus) equipped with an Olympus DFPL 2X-4 objective lens (numerical aperture 0.2) connected to a digital camera (Olympus E410) and flexible cold light (KL200; Olympus).

\section{Multiple myeloma xenograft model using the CAM assay}

The chick chorioallantoic membrane (CAM) assay is an established in vivo model that enables the investigation of tumor angiogenesis, metastasis and invasion without the need to sacrifice mature animals $[21,22]$. In brief, fertilized white Leghorn chicken eggs (SPF eggs; each group $n=5$ ) were purchased from Charles River and cultivated in an egg incubator at $37^{\circ} \mathrm{C}$ and $70 \%$ humidity (Compact S84) for three days. Thereafter, embryos were transferred to a plastic dish and incubated "ex ovo" for further four days, so that the CAM was able to develop. OPM- $2^{\text {eGFP }}$ and RPMI-8226 $6^{\text {eGFP }}$ myeloma cells $\left(2.5 \times 10^{5}\right)$ were mixed with rat-tail collagen and human bone-marrow mesenchymal stromal cells $\left(0.5 \times 10^{5}\right)$ and the $1 \mathrm{nM}$ of the respective compounds. Collagen drops were placed on parafilm for 30 min to allow polymerization of the extracellular matrix at $37^{\circ} \mathrm{C}$. Then onplants were transferred to the CAM of 7-day-old chicken embryos. After five days of in vivo growth, onplants were documented with the Olympus SZX10 stereomicroscope (Olympus) equipped with an Olympus DFPL 2X-4 objective lens (numerical aperture 0.2 ) connected to a digital camera (Olympus E410) and flexible cold light (KL200; Olympus). 


\section{Quantification of eGFP protein by ELISA}

MM spheroids or excised xenografts were transferred to $0.5 \mathrm{ml}$ RIPA Buffer (Sigma Aldrich) and homogenized with an Ultra Turrax Homogenizer three times for $5 \mathrm{sec}$ each on ice. Thereafter, homogenate underwent three freezing/thawing cycles in liquid nitrogen and a $37^{\circ} \mathrm{C}$ water bath. After centrifugation supernatants were diluted in assay buffer. GFP levels were measured with GFP ELISA Kit (Cell Biolabs) using biotinylated anti-GFP antibodies according to the manufacturer's protocol.

\section{The gelatin sponge-chorioallantoic membrane assay}

Fertilized white Leghorn chicken eggs were incubated at $37^{\circ} \mathrm{C}$ and constant humidity [23]. On day 3 , the shell was opened and $2 \mathrm{~mL}$ to $3 \mathrm{~mL}$ of albumen was removed to detach the chorioallantoic membrane (CAM). On day 8 , the CAMs were implanted with $1-\mathrm{mm}^{3}$ sterilized gelatin sponges (Gelfoam Upjohn) loaded with serum-free medium (SFM) alone (negative control) or CM of multiple myeloma endothelial cells (isolated accordingly Vacca et al. [24] alone (positive control) or with $0.1 \mathrm{nM}$ of each compound tested. The angiogenic response was evaluated on day 12 as the number of vessels converging toward the sponge at a magnification of $50 \mathrm{X}$ and photographed in ovo (Olympus stereomicroscope).

\section{Statistical analysis}

Statistical analyses were performed with the GraphPad Prism ${ }^{\mathrm{TM}}$ software for Windows. All tests of statistical significance were two-sided. Student's t-test, two-way ANOVA and Mann-Whitney U tests were used to study differences between the two groups.

\section{Authors' contributions}

Author's contributions to this work: EG, GU, JK and NS designed the study; GU and DR developed the methodology; NS, KJ, and CM performed the research; GU, NS, KJ, JK and CM analyzed the data; EG, GU and NS wrote the paper; MA, LFGF and WW gave administrative and material support, GG revised the manuscript.

\section{ACKNOWLEDGMENTS}

The authors thank Cornelia Heis and Silvia Karbon for their excellent technical assistance.

\section{Financial support}

This work was supported by the European Union Seventh Framework Program OPTATIO (FP7/2007- 2013) under grant agreement $n^{\circ} 278570$ and was performed in the framework of NS's $\mathrm{PhD}$ program for Clinical Cancer Research. JK was supported by the Competence Center ONCOTYROL and funded within the scope of the COMET - Competence Centers for Excellent Technologies. The COMET program is conducted by the Austrian Research Promotion Agency (FFG).

\section{CONFLICTS OF INTEREST}

MA and LFGF are employees of PharmaMar. All other authors declare no conflict of interest.

\section{REFERENCES}

1. Ferlay J, Steliarova-Foucher E, Lortet-Tieulent J, Rosso S, Coebergh JW, Comber H, Forman D, Bray F. Cancer incidence and mortality patterns in Europe: estimates for 40 countries in 2012. Eur J Cancer. 2013; 49: 1374-1403.

2. Lozano R, Naghavi M, Foreman K, Lim S, Shibuya K, Aboyans V, Abraham J, Adair T, Aggarwal R, Ahn SY, Alvarado M, Anderson HR, Anderson LM, et al. Global and regional mortality from 235 causes of death for 20 age groups in 1990 and 2010: a systematic analysis for the Global Burden of Disease Study 2010. Lancet. 2012; 380: 2095-2128.

3. Strobeck M. Multiple myeloma therapies. Nat Rev Drug Discov. 2007; 6: 181-182.

4. Kumar SK, Dispenzieri A, Lacy MQ, Gertz MA, Buadi FK, Pandey S, Kapoor P, Dingli D, Hayman SR, Leung N, Lust J, McCurdy A, Russell SJ, et al. Continued improvement in survival in multiple myeloma: changes in early mortality and outcomes in older patients. Leukemia. 2014; 28: 11221128.

5. Jurczyszyn A, Legiec W, Helbig G, Hus M, KyrczKrzemien S, Skotnicki AB. New drugs in multiple myeloma - role of carfilzomib and pomalidomide. Contemp Oncol (Pozn). 2014; 18: 17-21.

6. Kuehl WM, Bergsagel PL. Multiple myeloma: evolving genetic events and host interactions. Nat Rev Cancer. 2002; 2: $175-187$.

7. Prideaux SM, Conway O'Brien E, Chevassut TJ. The genetic architecture of multiple myeloma. Adv Hematol. 2014; 2014: 864058.

8. Kriangkum J, Motz SN, Debes Marun CS, Lafarge ST, Gibson SB, Venner CP, Johnston JB, Belch AR, Pilarski LM. Frequent occurrence of highly expanded but unrelated B-cell clones in patients with multiple myeloma. PLoS One. 2013; 8: e64927.

9. Garcia-Fernandez LF, Losada A, Alcaide V, Alvarez AM, 
Cuadrado A, Gonzalez L, Nakayama K, Nakayama KI, Fernandez-Sousa JM, Munoz A, Sanchez-Puelles J. Aplidin induces the mitochondrial apoptotic pathway via oxidative stress-mediated JNK and p38 activation and protein kinase C delta. Oncogene. 2002; 21: 7533-7544.

10. Caers J, Menu E, De Raeve H, Lepage D, Van Valckenborgh E, Van Camp B, Alvarez E, Vanderkerken $\mathrm{K}$. Antitumour and antiangiogenic effects of Aplidin in the 5TMM syngeneic models of multiple myeloma. Br J Cancer. 2008; 98: 1966-1974.

11. Broggini M, Marchini SV, Galliera E, Borsotti P, Taraboletti G, Erba E, Sironi M, Jimeno J, Faircloth GT, Giavazzi R, D'Incalci M. Aplidine, a new anticancer agent of marine origin, inhibits vascular endothelial growth factor (VEGF) secretion and blocks VEGF-VEGFR-1 (flt-1) autocrine loop in human leukemia cells MOLT-4. Leukemia. 2003; 17: 5259.

12. Ocio EM, Maiso P, Chen X, Garayoa M, Alvarez-Fernandez S, San-Segundo L, Vilanova D, Lopez-Corral L, Montero JC, Hernandez-Iglesias T, de Alva E, Galmarini C, Aviles $\mathrm{P}$, et al. Zalypsis: a novel marine-derived compound with potent antimyeloma activity that reveals high sensitivity of malignant plasma cells to DNA double-strand breaks. Blood. 2009; 113: 3781-3791.

13. Erba E, Bergamaschi D, Ronzoni S, Faretta M, Taverna S, Bonfanti M, Catapano CV, Faircloth G, Jimeno J, D'Incalci M. Mode of action of thiocoraline, a natural marine compound with anti-tumour activity. Br J Cancer. 1999; 80: 971-980.

14. Urdiales JL, Morata P, Nunez De Castro I, Sanchez-Jimenez F. Antiproliferative effect of dehydrodidemnin B (DDB), a depsipeptide isolated from Mediterranean tunicates. Cancer Lett. 1996; 102: 31-37.

15. Andersen RJ, Faulkner DJ, He CH, Van Duyne GD, Clardy J. Metabolites of the marine prosobranch mollusk Lamellaria sp. Journal of the American Chemical Society. 1985; 107: 5492-5495.

16. Davis RA, Carroll AR, Pierens GK, Quinn RJ. New lamellarin alkaloids from the australian ascidian, didemnum chartaceum. J Nat Prod. 1999; 62: 419-424.

17. Faircloth G, Cuevas C. Kahalalide F and ES285: potent anticancer agents from marine molluscs. Prog Mol Subcell Biol. 2006; 43: 363-379.

18. Fontana A, Cavaliere P, Wahidullah S, Naik CG, Cimino G. A new antitumor isoquinoline alkaloid from the marine nudibranch Jorunna funebris. Tetrahedron. 2000; 56: 73057308.

19. Frincke JM, Faulkner DJ. Antimicrobial metabolites of the sponge Reniera sp. Journal of the American Chemical Society. 1982; 104: 265-269.

20. Romero F, Espliego F, Perez Baz J, Garcia de Quesada T, Gravalos D, de la Calle F, Fernandez-Puentes JL. Thiocoraline, a new depsipeptide with antitumor activity produced by a marine Micromonospora. I. Taxonomy, fermentation, isolation, and biological activities. J Antibiot (Tokyo). 1997; 50: 734-737.

21. Deryugina EI, Quigley JP. Chick embryo chorioallantoic membrane model systems to study and visualize human tumor cell metastasis. Histochem Cell Biol. 2008; 130: 1119-1130.

22. Deryugina EI, Quigley JP. Chapter 2. Chick embryo chorioallantoic membrane models to quantify angiogenesis induced by inflammatory and tumor cells or purified effector molecules. Methods Enzymol. 2008; 444: 21-41.

23. Ribatti D, Nico B, Vacca A, Presta M. The gelatin spongechorioallantoic membrane assay. Nat Protoc. 2006; 1: 8591.

24. Vacca A, Ria R, Semeraro F, Merchionne F, Coluccia M, Boccarelli A, Scavelli C, Nico B, Gernone A, Battelli F, Tabilio A, Guidolin D, Petrucci MT, et al. Endothelial cells in the bone marrow of patients with multiple myeloma. Blood. 2003; 102: 3340-3348. 